\title{
Optimization of Biodiesel Production from African Crude Palm Oil (Elaeis guineensis Jacq) with High Concentration of Free Fatty Acids by a Two-Step Transesterification Process
}

\author{
Francisco Anguebes-Franseschi1 ${ }^{*}$, Atl Córdova-Quiroz ${ }^{1}$, Julia Cerón-Bretón'1, \\ Claudia Aguilar-Ucan'1, Gloria Castillo-Martínez², Rosa Cerón-Bretón'1, \\ Alejandro Ruíz-Marín1, Carlos Montalvo-Romero1 \\ ${ }^{1}$ Facultad de Química, Universidad Autónoma del Carmen, Ciudad del Carmen, México \\ ${ }^{2}$ Departamento de Informática, Instituto Tecnológico de Villahermosa, Villahermosa, México \\ Email: ${ }^{*}$ fanguebes@pampano.unacar.mx
}

Received 10 November 2015; accepted 11 January 2016; published 14 January 2016

Copyright (C) 2016 by authors and Scientific Research Publishing Inc.

This work is licensed under the Creative Commons Attribution International License (CC BY).

http://creativecommons.org/licenses/by/4.0/

(c) (i) Open Access

\section{Abstract}

In this study, African crude palm olein (CPO) was used to synthesize biodiesel. The objective was to determine the optimal reaction conditions for the methanolysis of olein. The used CPO had a $5.72 \%$ concentration of free fatty acids (FFA); thus, the production of biodiesel was carried out in two stages: 1) esterification using sulfuric acid and 2) transesterification using sodium methoxide. In order to optimize the yield of biodiesel during the transesterification process, a central rotatable design and the response surface methodology were used. The studied variables were: catalyst loading, reaction time and reaction temperature. The analysis of variance showed that the variables with significant effect were the catalyst loading, reaction temperature and reaction time; as well as the catalyst loading*reaction temperature and catalyst loading*reaction time. Results indicate that the optimal reaction conditions during transesterification are: $0.65 \%$ catalyst loading ( $w t / w t_{\text {aceite) }}$, reaction time of $135 \mathrm{~min}$ and a reaction temperature of $56^{\circ} \mathrm{C}$. The optimal reaction conditions during esterification are: $2.5 \%$ weight of catalyst, reaction time of $150 \mathrm{~min}$ and a reaction temperature of $64.5^{\circ} \mathrm{C}$. Under these conditions, a $90 \%$ yield of biodiesel was obtained.

\footnotetext{
"Corresponding author.
} 


\section{Keywords}

\section{Acid Catalysis, Alkaline Catalysis, Biodiesel Process Optimization, Response Surface Methodology}

\section{Introduction}

Biodiesel is a biofuel obtained from the transesterification of vegetable oil or animal fat with a low molecular weight alcohol under the presence of a catalyst. The most used ones are sodium or potassium hydroxide due to their low cost and high efficiency [1]. Biodiesel may be used in pure form or mixed with petrodiesel, as a total or partial substitute of diesel oil in internal combustion engines [2]. This offers various advantages over petrodiesel, i.e. it is biodegradable, it is obtained from renewable materials, it has a cleaner combustion due to its low contaminant emissions, it is non-toxic, and it provides a good lubrication of engine parts, among others. From the social point of view, biodiesel may produce socioeconomic benefits, i.e. added value to raw material, generation of rural jobs and employments for the manufacturing and distribution of biodiesel [3].

In order to improve the economic feasibility of biodiesel production, low cost feedstock such as animal fats, waste cooking oil, raw oil such as crude palm olein (CPO) and Jatrophacurcas oil, are promising to replace refined vegetables oils [4]. One of the most potential plant species for the production of oil in the world is the African oil palm (Elaeis guineensis Jacq). Its cultivation has widely spread due to its adaptation and high yields up to $5.5 \mathrm{t}^{\mathrm{h}} \mathrm{ha}^{-1}$ of oil [5]. Production of the oil palm as bioenergetic has increased significantly because of its low cost. Other advantages of African oil palm plantations is their great capacity for $\mathrm{CO}_{2}$ capture (up to 4.8 $\mathrm{t} \cdot \mathrm{ha}^{-1}$ per year); therefore, resulting in an environmental benefit. It has also been proven that byproducts of oil palm can be used for the generation of electricity [6].

One important chemical property to be quantified in crude oil palm (CPO) is the free fatty acid (FFAs) contents, as this property may aid in determining the type of process to be followed for obtaining biodiesel. The basic catalysis is not recommended in the case of oils with a high free fatty acid concentration $(>0.5 \%)$, due to the formation of soap, leading to a decrease in the yield of biodiesel and a considerable increase in the production costs. That is why it is necessary to apply a pre-treatment (esterification) with an acidic catalyst in order to reduce the FFAs concentration to less than $0.5 \%$ [7]. Several researches propose a two-step acid-alkaline catalyzed process [8].

The African palm cultivation in Mexico is carried out in the states of Chiapas, Veracruz, Tabasco and Campeche, with 54,434 ha planted in 2010; but there is potential to grow up to 2.5 million ha, and 400,000 of them in the state of Tabasco. The oil palm represented a potential to make biodiesel in México [9]. The goal of this study was to determine the optimal reaction conditions for themethanolysis of crude palm olein at the laboratory scale.

\section{Materials and Methods}

All reagents used during the development of this research were reagent grade compounds (methanol, sodium hydroxide analytical grade and sulfuric acid) acquired from Fermont. The FAME Standard Supelco, USA was used to perform thecalibration curve. A commercial grade CPO from Agroindustrias de Palenque S.A. de C.V. was used, with a concentration of $5.72 \mathrm{wt} \%$ of FFAs. Therefore, a pre-treatment was needed in order to reduce below $0.5 \%$ the concentration of FFA.

\section{Experimental Design}

The synthesis of biodiesel from palmoil transesterification using oil palm was developed and optimized using the Central Composite Design (CCD) and Response Surface Methodology (RSM). CCD helps in investigating linear, quadratic, and cross-product effects of the three reaction condition variables on the biodiesel yield. The three independent variables studied were catalyst loading, reaction temperature and reaction time, temperature Table 1 lists the range and levels of the three independent variables studied. Selection of the levels was carried out on the basis of results obtained in a preliminary study, considering limits for the experiment set-up and 
Table 1. Factors and levels employed for the response surface design.

\begin{tabular}{ccccccc}
\hline & \multicolumn{7}{c}{ Levels } \\
\hline Variable & Coding & $-1.68(-\alpha)$ & -1 & 0 & 1 & $+1.68(+\alpha)$ \\
\hline Catalyst loading $(\mathrm{wt} \%)$ & $X_{1}$ & 0.26 & 0.4 & 0.6 & 0.8 & 0.96 \\
Reaction temperature $\left({ }^{\circ} \mathrm{C}\right)$ & $X_{2}$ & 46.6 & 50 & 55 & 60 & 63.4 \\
Reaction time $(\mathrm{min})$ & $X_{3}$ & 46 & 60 & 80 & 100 & 114 \\
\hline
\end{tabular}

working conditions for each chemical species. The complete design matrix of the experiments employed and their results are given in Table 2. All variables at the zero level constitute the center points and the combination of each of the variables at either it is lowest $(-1.68)$ level or highest $(+1.68)$ level with the order variables at zero level constitute the axial points. Twenty tests were performed with a threefold repetition, eight factorials $\left(2^{3}\right)$, six axial and six central (Table 2) [10] [11]. The experiment sequence was randomized to minimize the effects of the uncontrolled factors. The depend variable $(Y)$ which corresponds to the yield of methyl esters and which is calculated with Equation (1) [11].

$$
Y=[(\text { mass of methyl ester produced }(\mathrm{g})) /(\text { mass of oil used }(\mathrm{g}))] \times 100
$$

\section{Experimental Procedure}

\subsection{Esterification}

The CPO esterification was performed in a three-neck flask connected to a thermometer, a reflux condenser. The three-neck batch reactor was put in a temperature controlled jacket over a magnetic stirrer/hot plate (controlled Barnstead-Electrothermal BI, USA), which could provide good temperature control within $1^{\circ} \mathrm{C}$ Figure 1 . In this work in total $4 \mathrm{~kg}$ (CPO) was used for the acid-catalyzed pre-esterification. In the methodological development; first $500 \mathrm{~g}$ of CPO carefully transferred into the reaction flask and preheated to $60^{\circ} \mathrm{C}$. For acid-catalyzed esterification trials, sulfuric acid $\left(\mathrm{H}_{2} \mathrm{SO}_{4}\right)$ was used as catalyst. The sulfuric acid and methanol solution were freshly prepared in a $500 \mathrm{~mL}$ Erlenmeyer flask admixing methanol 20\% w/w and sulfuric acid 2.5\% w/w based on the weight of CPO. The mixture sulfuric acid and methanol were added to the CPO into reaction flask. The reaction mixture was heated up to the boiling point of methanol $\left(64.5^{\circ} \mathrm{C}\right)$ and the esterification reaction was taken for 150 min at atmospheric pressure. With the aim of promoting mass transfer among the reagents was mixing with magnetic stirring. At the completion of the 150 min of reaction, the mixture was poured in a separator funnel, the mixture components form two immiscible phases. The upper phase containing methanol and water; the bottom phase contained the esterified palm olein (EPO). The EPO was decanted by gravity, packaged into amber colored bottles and maintained in refrigeration at $4{ }^{\circ} \mathrm{C}$ until its use in the transesterification stage. The analysis showed that the EPO had a $0.45 \%$ concentration of FFA, which allows using basic catalysis for the transesterification stage.

\subsection{Transesterification}

For this stage, $150 \mathrm{~g}$ of EPO were weighed in $250 \mathrm{~mL}$ Erlenmeyer flask and it was preheated at the test temperature. In another similar flask catalyst (sodium methoxide) was prepared by reaction of anhydrous methanol with $\mathrm{NaOH}$; the mixture was stirred until the hydroxide total dilution. EPO was transferred to the reactor and then to catalyst, the mixture was heated to the temperature for each treatment (Table 2). After the reaction, the mixture was poured in a separating funnel and washed with deionized water; at this stage two immiscible phases were formed. The aqueous phase contained glycerol, methanol, soap and catalyst residues which were decanted by gravity. The upper phase contained biodiesel was filtered through Whatman \# 40 filter paper to remove residual soap and catalyst. The resulting biodiesel was weighed to calculate the yield. The biodiesel was refined through a glass column packed with $60 \mathrm{~g}$ of Amberlite BD10. Aliquots were taken for subsequent chromatographic analysis.

\subsection{Statistical Analysis}

The statistical data was analyzed with the response surface methodology by means of the (STATISTICA V 7 software). A second order polynomial was used to predict the yield of biodiesel synthesized from the transesteri- 


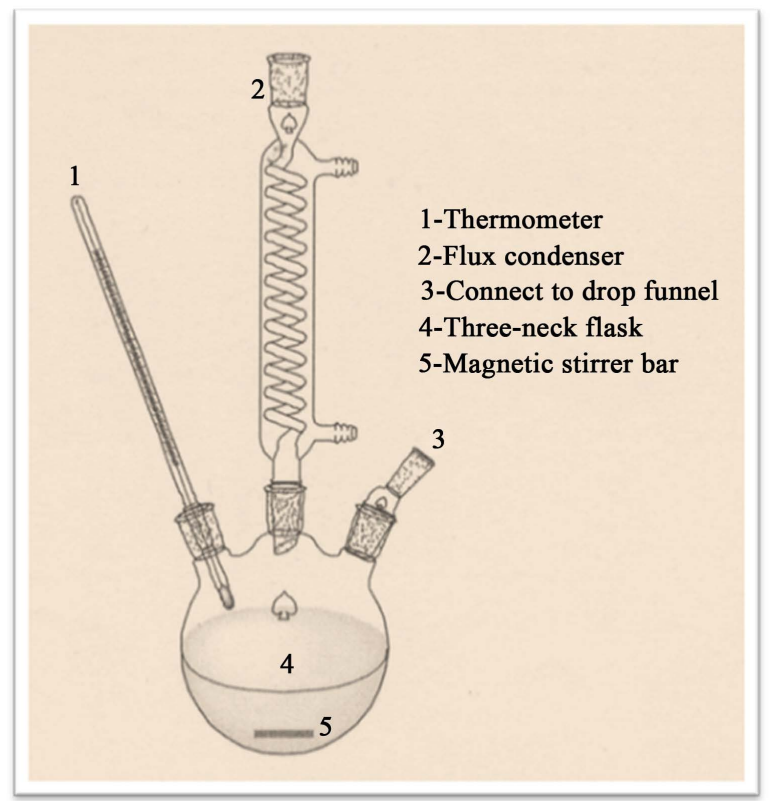

Figure 1. Schematic diagram of reactor.

Table 2. Second order central composite rotatable design.

\begin{tabular}{|c|c|c|c|c|c|}
\hline Run & $X_{1}$ & $X_{2}$ & $X_{3}$ & $Y_{\text {exp. }}$ & $Y_{\text {model }}$ \\
\hline 1 & 0.4 & 50 & 60 & 68.96 & 68.44 \\
\hline 2 & 0.8 & 50 & 60 & 74.52 & 74.65 \\
\hline 3 & 0.4 & 60 & 60 & 71.39 & 71.63 \\
\hline 4 & 0.8 & 60 & 60 & 73.98 & 74.91 \\
\hline 5 & 0.4 & 50 & 100 & 74.13 & 74.11 \\
\hline 6 & 0.8 & 50 & 100 & 82.57 & 83.17 \\
\hline 7 & 0.4 & 60 & 100 & 77.18 & 77.88 \\
\hline 8 & 0.8 & 60 & 100 & 82.57 & 84.01 \\
\hline 9 & 0.26 & 55 & 80 & 57.38 & 58.23 \\
\hline 10 & 0.96 & 55 & 80 & 64.91 & 65.97 \\
\hline 11 & 0.6 & 46.6 & 80 & 78.82 & 80.45 \\
\hline 12 & 0.6 & 63.4 & 80 & 83.57 & 83.45 \\
\hline 13 & 0.6 & 55 & 46 & 76.41 & 77.95 \\
\hline 14 & 0.6 & 55 & 114 & 90.15 & 90.501 \\
\hline 15 & 0.6 & 55 & 80 & 88.57 & 88.71 \\
\hline 16 & 0.6 & 55 & 80 & 87.13 & 88.71 \\
\hline 17 & 0.6 & 55 & 80 & 89.02 & 88.71 \\
\hline 18 & 0.6 & 55 & 80 & 86.94 & 88.71 \\
\hline 19 & 0.6 & 55 & 80 & 88.78 & 88.71 \\
\hline 20 & 0.6 & 55 & 80 & 87.97 & 88.71 \\
\hline
\end{tabular}


fied palm olein EPO, Equation (2) [12].

$$
y=\beta_{0}+\sum_{i=1}^{k} \beta_{i} x_{i}+\sum_{i=1}^{k} \beta_{i i} x_{i}^{2}+\sum_{i>j}^{k} \sum_{j=1}^{k} \beta_{i j} x_{i} x_{j}+e
$$

where $y$ is the response variable (yield of biodiesel, in weight percent); $X_{i}$ and $X_{j}$ are independent variables; $\beta_{0}, \beta_{i}$, $\beta_{i i}, \beta_{i j}$ are the model parameters; $e$ is the experimental error. The prediction capacity of the second order model was assessed through an analysis of variance. The effect of each factor and its interactions were determined with the value of the statistical $p$ for values of $(p<0.05)$. Further, the $p$ values were used to determine the significance of each coefficient and its respective interactions.

\subsection{Analytical Procedure}

The amount of methyl esters in the product of biodiesel CPO was analyzed on an Agilentgas chromatograph model 6890 coupled to a mass detector model 2973N, Agilent, equipped with a chromatographic column HP 5MS 5\% dyphenyl-95\% dymethylsiloxane $(25 \mathrm{~m} \times 0.2 \mathrm{~mm} \times 0.33 \mu \mathrm{m})$ and ionization technique EI at $70 \mathrm{eV}$, was used to analyze the samples of biodiesel. Helium was used as carrier gas at a flow of $1 \mathrm{~mL} / \mathrm{min}$. The initial oven temperature was set at $40^{\circ} \mathrm{C}$ for 1 minute; the column temperature was set at $250^{\circ} \mathrm{C}$, the linear temperature ramp was set at a rate of $5^{\circ} \mathrm{C} \mathrm{min}{ }^{-1}$ up to $250^{\circ} \mathrm{C}$ and maintained at $250^{\circ} \mathrm{C}$ for $5 \mathrm{~min}$. The injector and the detector were maintained at $250^{\circ} \mathrm{C}$ and $280^{\circ} \mathrm{C}$ respectively. Approximately $2 \mu \mathrm{L}$ of the biodiesel products was dissolved with $500 \mu \mathrm{L}$ of dichloromethane, and $1 \mu \mathrm{L}$ of this mixture was injected into the chromatograph. The mass spectra were compared with the NIST data base (version 1.7a) in order to identify all components of biodiesel, as well as the relative abundance of the characteristic peaks of the compounds of interest to quantify the concentration.

\section{Results and Discussion}

The analysis of variance shows that the second order model effectively predicts the observed values as function of the studied factors. The statistical $p$ values, assessed with a $95 \%$ reliability, emphasize the variables that had a significant effect on the transesterification reaction, which were those having values of $p$ less than 0.05 (Table 3 ). Variables having a greater contribution on the yield of biodiesel were: catalyst loading, reaction temperature and reaction time, the interactions catalyst loading*reaction temperature and catalyst loading*reaction time.

Mathematical model was generated in function of the variables having significant effect (Equation (3)). This model was used to predict the values of $Y$ under certain reaction conditions. The coefficient of determination between the observed and predicted values was highly significant $\left(R^{2}=97.28 \%\right)$ and indicates that a high proportion of the response is attributable to three predictive variables considered, whereas a $2.72 \%$ variability in the response is attributable to non-controlled factors.

$$
\begin{aligned}
Y= & -340.355+303.461 X_{1}-218.288 X_{1}^{2}+10.753 X_{2}-0.09298 X_{2}^{2}+0.6191 X_{3} \\
& -0.00388 X_{3}^{2}-0.7525 X_{1} X_{2}+0.1775 X_{1} X_{3}+0.00145 X_{2} X_{3}
\end{aligned}
$$

\section{Effect of Reaction Parameters}

The analysis of variance shows that the factors having a greater contribution to the yield of methyl ester are catalyst loading and the reaction temperature Table 3. A simultaneous increase in the catalyst loading from $0.26 \mathrm{wt} \%$ and $46.6^{\circ} \mathrm{C}$ for the reaction temperature has a positive effect on the yield of biodiesel (Figure 2), which is attributed to the temperature increment and the reaction rate between the reactants. The contour plot (Figure 2) shows the maximum yield of methyl ester, about $90 \mathrm{wt} \%$, was reached at a concentration of $0.65 \mathrm{wt} \%$ for the catalyst loading and $56^{\circ} \mathrm{C}$ for the reaction temperature, and 114 min of reaction time, see Table 2 (run 14). For catalyst loading values and reaction temperature above these values, the effect of such factors was negative on the yield of biodiesel; in as much as said conditions promote the saponification of triglycerides. The saponification and transesterification reactions occur simultaneously, wherein the triglycerides react with the catalyst to produce sodium soaps. The presence of soap increases the viscosity of the reagents, thus becoming difficult to purify the biodiesel. Such factors contribute to a reduction in yield of methyl esters. The aforementioned behavior was previously studied [13] in the transesterification of soybean, and castor oil; and in the transesterification of Brassica carinata oil [14] [15]. 


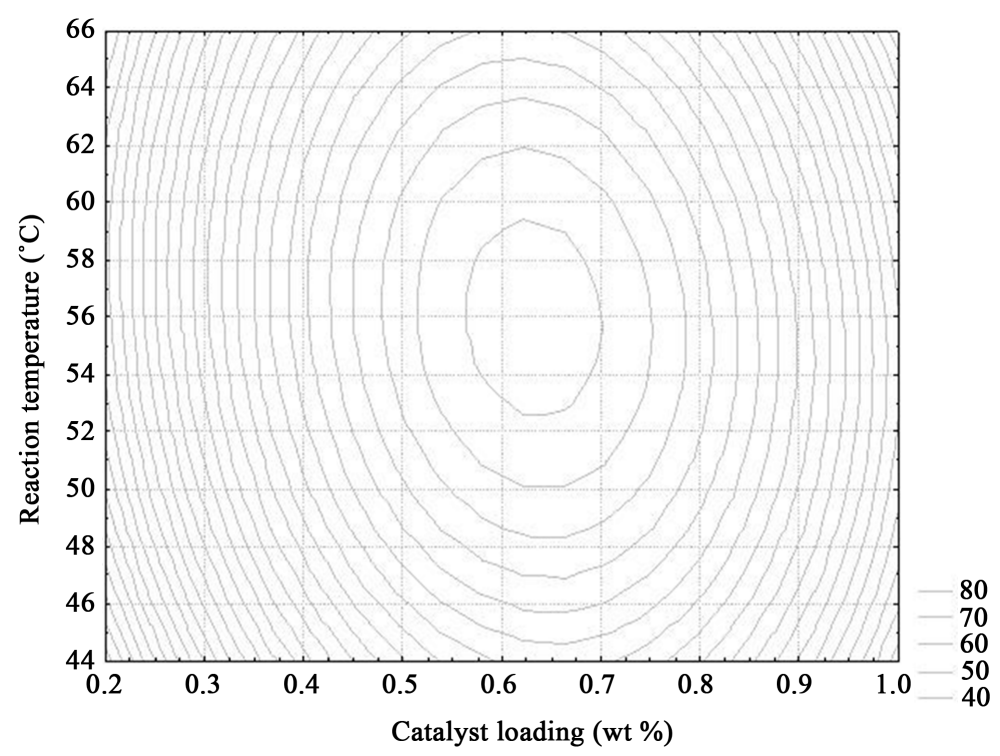

Figure 2. Contour plot shows the yield of biodiesel (wt\%) as function of the catalyst loading and reaction temperature.

Table 3. Analysis of variance for response surface of the second order model.

\begin{tabular}{|c|c|c|c|c|c|}
\hline Effect & SS & Degrees of freedom & MS & $\mathrm{F}$ & $p$ \\
\hline Model & 78.093 & 1 & 78.093 & 94.629 & 0.000002 \\
\hline$X_{1}$ & 189.535 & 1 & 189.535 & 229.668 & 0 \\
\hline$X_{1}^{2}$ & 1250.05 & 1 & 1250.05 & 1514.743 & 0 \\
\hline$X_{2}$ & 78.029 & 1 & 78.029 & 94.552 & 0.000002 \\
\hline$X_{2}^{2}$ & 77.268 & 1 & 77.268 & 93.63 & 0.000002 \\
\hline$X_{3}$ & 7.432 & 1 & 7.432 & 9.006 & 0.01332 \\
\hline$X_{3}^{2}$ & 35.757 & 1 & 35.757 & 43.329 & 0.000062 \\
\hline$X_{1} X_{2}$ & 4.53 & 1 & 4.53 & 5.489 & 0.041134 \\
\hline$X_{1} X_{3}$ & 4.033 & 1 & 4.033 & 4.887 & 0.050509 \\
\hline$X_{2} X_{3}$ & 0.168 & 1 & 0.168 & 0.204 & 0.661291 \\
\hline Lack of fit & 8.253 & 10 & 0.825 & & \\
\hline
\end{tabular}

In the transesterification reaction four reversible complex chemical reactions occur because are sensitive to variations in the catalyst loading, alcohol, and the temperature and time reaction. These variations can significantly affect chemical equilibrium, and increase or decrease the performance of methyl esters [16]. The simultaneous effect of the catalyst loading and the reaction time is presented in Figure 3. As shown methyl esters performance increases with increasing catalyst loading and reaction time; for a reaction time of 110 min and $0.65 \mathrm{wt} \%$ of catalyst performance is maximized biodiesel; or higher values to these performance decreases biodiesel which could be attributed to excess catalyst promote the saponification of triglycerides, and reversibility of transesterification reactions; this behavior was also reported in previous studies in the transesterification of canola oil [16]. The optimal conditions for the transesterification were: a catalyst loading 0.65 wt $\%$, reaction temperature of $56^{\circ} \mathrm{C}$ and reaction time of $110 \mathrm{~min}$ (Figure 1 and Figure 2).

Gas chromatography (GC) measurements of the biodiesel prepared CPO showed a combination of various types of methyl esters (Figure 4). Individual peaks in the GC results were analyzed and identified using mass 


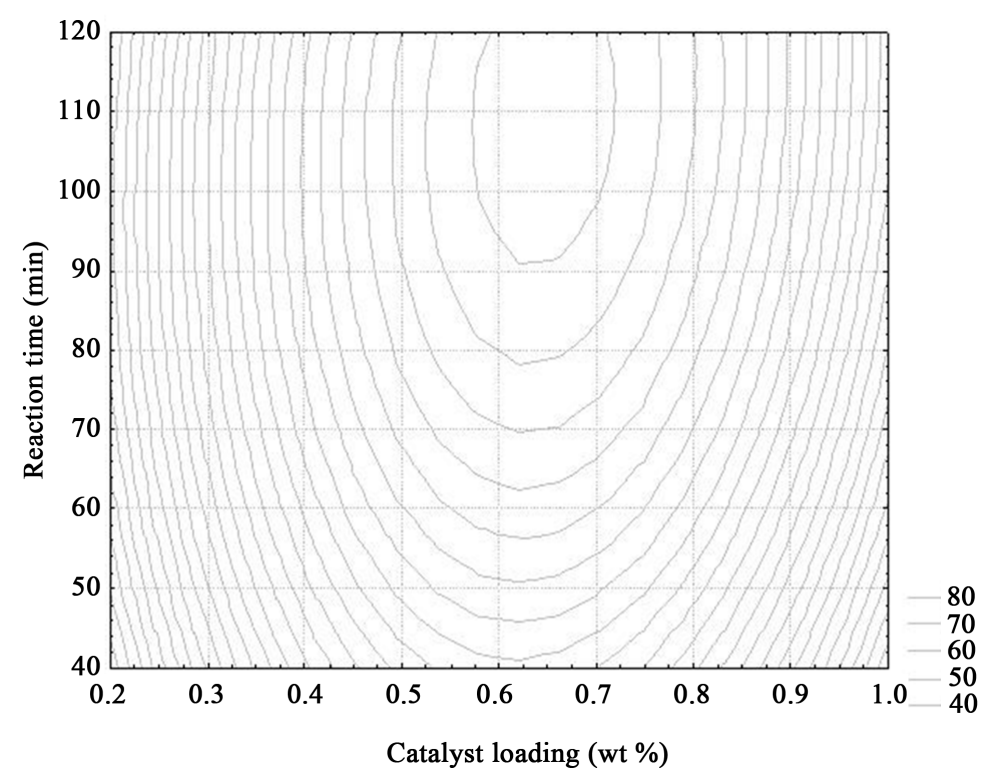

Figure 3. Contour plots shows the yield of biodiesel (wt\%) as function of the catalyst loading and reaction time.

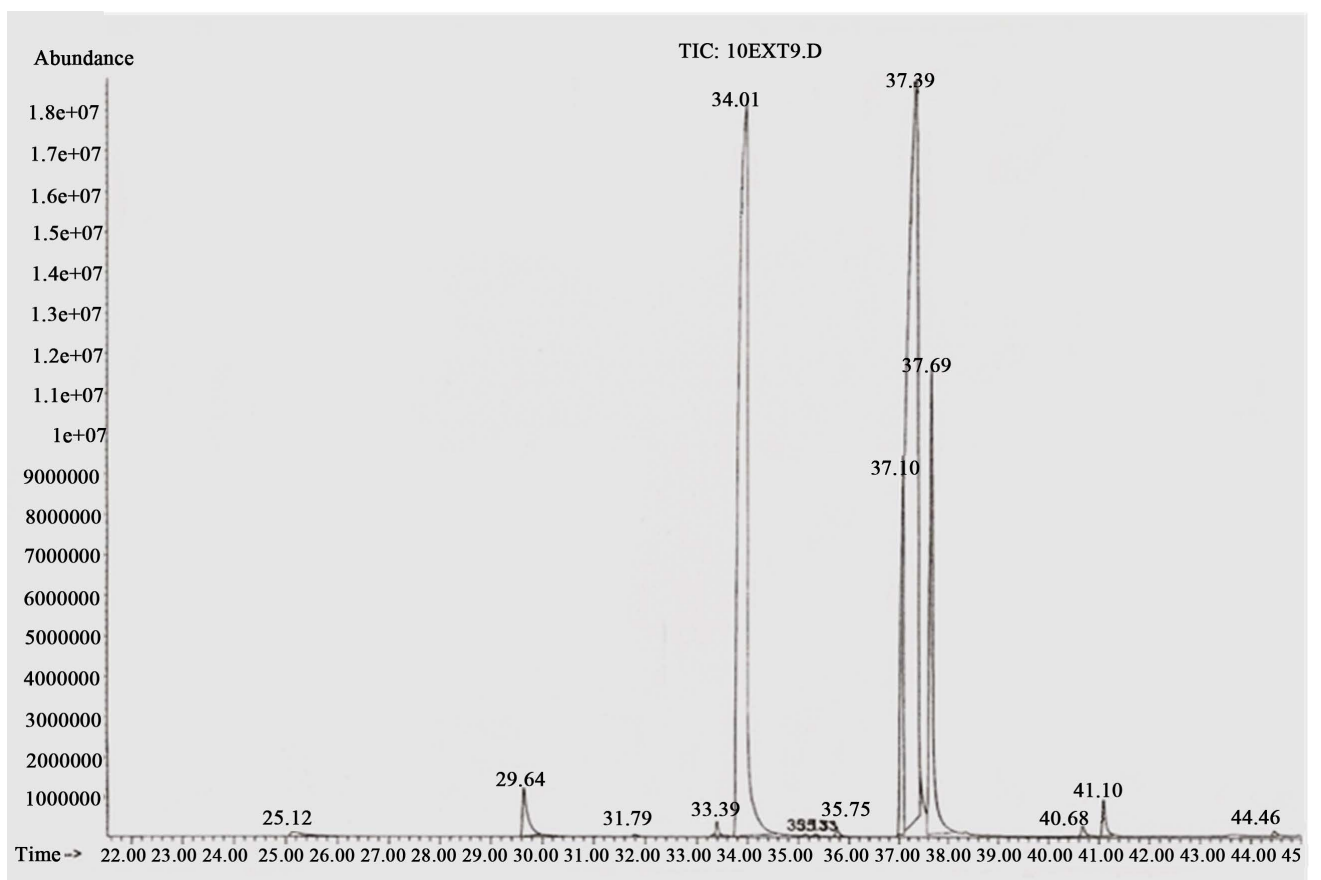

Figure 4. Gas chromatogram of the biodiesel showed different methyl esters of fatty acids present in the biodiesel produced from CPO, identification of individual methyl esters was achieved using mass spectroscopy (MS).

spectroscopy (MS). It is noted that feather meal biodiesel contains a wide variety of fatty acid methyl esters ranging from $\mathrm{C} 12$ to $\mathrm{C} 22$. The first compound, identified at $34 \mathrm{~min}$, corresponds to methyl hexadecanoate with a concentration of $39.1 \mathrm{wt} \%$; the compound identified at $37.1 \mathrm{~min}$ is methyl 9.12-octadecadienoate with 5.755 wt\%; the compound with the highest concentration was identified at 37.397 min corresponding to methyl 9-octadecenoate with $44.378 \mathrm{wt} \%$; the component identified at $37.68 \mathrm{~min}$ is methyl octadecanoate with $9.592 \mathrm{wt} \%$. The composition of feather meal biodiesel is mentioned in Table 4. Biodiesel containing compounds with carbon chain lengths $\geq 15$ produces superior-quality fuel. The presence of saturated fatty acids (50 wt\%) gave a good oxidative stability to biodiesel. 
Table 4. Fatty acid profile of biodiesel produced from CPO calculated from GC-MS.

\begin{tabular}{ccc}
\hline Components & Retention time (min) & Weight (\%) \\
\hline Dodecanoate of methyl & 25.12 & 0.293 \\
Tetradecanoate of methyl & 29.64 & 1.357 \\
Pentadecanoate of methyl & 31.79 & 0.049 \\
9-Hexadecenoate of methyl & 33.39 & 0.209 \\
Hexadecanoate of methyl & 34.01 & 37.109 \\
Cyclepropanoctanoate de 2-hexylmethyl & 35.53 & 0.037 \\
Heptadecanoato de metilo & 35.75 & 0.171 \\
9-12-Octadecadienoate of methyl & 37.100 & 5.755 \\
9-Octadecenoate of methyl & 37.39 & 44.378 \\
Octadecanoate of methyl & 37.69 & 9.592 \\
11-Eicosenoate of methyl & 40.66 & 0.215 \\
Eicosanoate of methyl & 41.10 & 0.702 \\
Docosanoate of methyl & 44.46 & 0.101
\end{tabular}

\section{Conclusion}

In this work, a two-step homogeneous catalyzed CPO transesterification reaction for biodiesel production was investigated. The statistical significant variables, their effects and the higher order interaction effects on process efficiency were identified. The results show that the response surface methodology and central composite rotatable design are useful in order to determine the effect of the catalyst loading, temperature and reaction time on the transesterification reaction of African crude palm olein (CPO). This allowed finding the optimal values of the process variables maximizing the yield of biodiesel. Analysis of variance shows that the variables having a significant effect on the yield of biodiesel were: catalyst loading $\left(X_{1}\right)$, reaction temperature $\left(X_{2}\right)$ and reaction time $\left(X_{3}\right)$, as well as the interactions catalyst loading*reaction temperature and catalyst loading*reaction time. The optimal conditions for esterification were $2.5 \mathrm{wt} \%$ concentration of sulfuric acid, reaction time of $150 \mathrm{~min}$ and temperature of $64.5^{\circ} \mathrm{C}$, under these reaction conditions, the concentration of FFAs decreases from 5.72 to 0.45 wt $\%$, allowing the use of alkaline catalyst. The optimal conditions for the transesterification were: catalyst loading 0.65 wt $\%$, reaction temperature of $56^{\circ} \mathrm{C}$ and reaction time of $114 \mathrm{~min}$ and methanol concentration of $20 \mathrm{wt} \%$. The results show that it is feasible to produce biodiesel by using African crude palm olein as low cost raw material through a two-step process: acid esterification and alkaline transesterification allowing biodiesel yield near of $90 \mathrm{wt} \%$, with a low energy cost and overall reaction time of $264 \mathrm{~min}$.

\section{Acknowledgements}

The authors are grateful to the PROMEP project PROMEP/103.5/09/4363, to the Universidad Autónoma del Carmen.

\section{Conflict of Interest}

The authors declare not have interest conflicts.

\section{References}

[1] Haas, M.J., Scott, K.M., Marmer, W.N. and Foglia, T.A. (2004) In Situ Alkaline Transesterification: An Effective Method for the Production of Fatty Acid Esters from Vegetable Oils. Journal of the American Oil Chemists' Society, 81, 
83-89. http://dx.doi.org/10.1007/s11746-004-0861-3

[2] Teixeira, E., Mattiuzi, C., Feltes, S., Wiegand, F. and Santana, E. (2012) Estimated Atmospheric Emissions from Biodiesel and Characterization of Pollutants in the Metropolitan Area of Porto Alegre-RS. Anais da Academia Brasileira de Ciências, 84, 665-667. http://dx.doi.org/10.1590/S0001-37652012000300008

[3] Demirbas, A. (2007) Importance of Biodiesel as Transportation Fuel. Energy Police, 35, 4661-4670. http://dx.doi.org/10.1016/j.enpol.2007.04.003

[4] Pahn, A.N. and Pahn, T.M. (2008) Biodiesel Production from Waste Cooking Oils. Fuel, 87, 3490-3496. http://dx.doi.org/10.1016/j.fuel.2008.07.008

[5] Fargione, J.E., Plevin, R.J. and Hill, J.D. (2010) The Ecological Impact of Biofuels. Annual Review of Ecology, Evolution, and Systematics, 41, 351-377. http://dx.doi.org/10.1146/annurev-ecolsys-102209-144720

[6] Johnston, M. and Holloway, T. (2007) A Global Comparison of National Biodiesel Production Potentials. Environmental Science \& Technology, 41, 7967-7973.

[7] Marchetti, J.M. and Errazu, A.F. (2008) Esterification of Free Fatty Acids Using Sulfuric Acid as Catalyst in the Presence of Triglycerides. Biomass and Bioenergy, 32, 892-895. http://dx.doi.org/10.1016/j.biombioe.2008.01.001

[8] Wang, Y., Ou, S., Liu, P. and Zhang, Z. (2007) Preparation of Biodiesel from Waste Cooking Oil via Two Step Catalysed Process. Energy Conversion and Management, 48, 184-188. http://dx.doi.org/10.1016/j.enconman.2006.04.016

[9] Lozada, I., Islas, J. and Grande, G. (2010) Environmental and Economic Feasibility of Palm Oil Biodiesel in the Mexican Transportation Sector. Renewable and Sustainable Energy Reviews, 14, 486-492. http://dx.doi.org/10.1016/j.rser.2009.06.034

[10] Umer, R., Farooq, A., Tariq, M.A., Muhammad, A. and Mushtaq, A. (2009) Optimization of Alkaline Transesterification of Rice Bran Oil for Biodiesel Production Using Response Surface Methodology. Journal of Chemical Technology and Biotechnology, 84, 1364-1370. http://dx.doi.org/10.1002/jctb.2191

[11] Kafuku, G., Teong-Lee, K. and Mbarawa, M. (2010) The Use of Sulfated Tin Oxide as Solid Superacid Catalyst for Heterogeneous Transesterification of Jatrophacurcas Oil. Chemical Papers, 64, 734-740. http://dx.doi.org/10.2478/s11696-010-0063-1

[12] Gwi-Taek, J. and Park, D.H. (2009) Optimization of Biodiesel Production from Castor Oil Using Response Surface Methodology. Applied Biochemistry and Biotechnology, 156, 431-441.

[13] De Oliveira, D., Di Luccio, M., Faccio, C., Dalla-Rosa, C., Bender, J.P., Lipken, N., Amroginski, C., Dariva, C. and De Oliveira, J.V. (2005) Optimization of Alkaline Transesterification of Soybean Oil for Biodiesel Production. Applied Biochemistry and Biotechnology, 122, 553-560.

[14] Vicente, G., Martínez, M. and Aracil, J. (2005) Optimization of Brassica carinata Oil Methanolysis for Biodiesel Production. Journal of the American Oil Chemists' Society, 82, 899-904. http://dx.doi.org/10.1007/s11746-005-1162-6

[15] Aracil, J., Bouaid, A. and Martinez, M. (2007) A Comparative Study of Ethyl Esters from Vegetable Oils as a Biodiesel Fuel Optimization by Factorial Design. Chemical Engineering Journal, 134, 93-99. http://dx.doi.org/10.1016/j.cej.2007.03.077

[16] Joshi, H., Toler, J., Moser, B.R. and Walker, T. (2009) Biodiesel from Canola Oil Using a 1:1 Molar Mixture of Methanol and Ethanol. European Journal of Lipid Science and Technology, 111, 464-473.

http://dx.doi.org/10.1002/ejlt.200800071 